\title{
Investigating MicroRNA and transcription factor co-regulatory networks in colorectal cancer
}

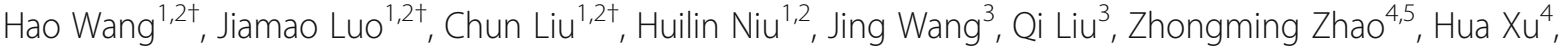 \\ Yanqing Ding ${ }^{1,2}$, Jingchun Sun ${ }^{4^{*}}$ and Qingling Zhang ${ }^{1,2^{*}}$
}

\begin{abstract}
Background: Colorectal cancer (CRC) is one of the most common malignancies worldwide with poor prognosis. Studies have showed that abnormal microRNA (miRNA) expression can affect CRC pathogenesis and development through targeting critical genes in cellular system. However, it is unclear about which miRNAs play central roles in CRC's pathogenesis and how they interact with transcription factors (TFs) to regulate the cancer-related genes.

Results: To address this issue, we systematically explored the major regulation motifs, namely feed-forward loops (FFLs), that consist of miRNAs, TFs and CRC-related genes through the construction of a miRNA-TF regulatory network in CRC. First, we compiled CRC-related miRNAs, CRC-related genes, and human TFs from multiple data sources. Second, we identified 13,123 3-node FFLs including 25 miRNA-FFLs, 13,005 TF-FFLs and 93 composite-FFLs, and merged the 3-node FFLs to construct a CRC-related regulatory network. The network consists of three types of regulatory subnetworks (SNWs): miRNA-SNW, TF-SNW, and composite-SNW. To enhance the accuracy of the network, the results were filtered by using The Cancer Genome Atlas (TCGA) expression data in CRC, whereby we generated a core regulatory network consisting of 58 significant FFLs. We then applied a hub identification strategy to the significant FFLs and found 5 significant components, including two miRNAs (hsa-miR-25 and hsa-miR-31), two genes (ADAMTSL3 and AXIN1) and one TF (BRCA1). The follow up prognosis analysis indicated all of the 5 significant components having good prediction of overall survival of CRC patients.
\end{abstract}

Conclusions: In summary, we generated a CRC-specific miRNA-TF regulatory network, which is helpful to understand the complex CRC regulatory mechanisms and guide clinical treatment. The discovered 5 regulators might have critical roles in CRC pathogenesis and warrant future investigation.

Keywords: Colorectal cancer (CRC), microRNA, Transcription factor, Feed-forward loops (FFLs), Regulatory network

\section{Background}

Colorectal cancer (CRC) is one of the most common malignant tumors in the human digestive system and has the third highest incidence and mortality of all malignancies [1-3]. Uncovering the regulation and progression mechanisms of CRC is important for developing effective molecular therapeutic strategies. In the last decades, substantial efforts have been made to collect

\footnotetext{
* Correspondence: jingchun.sun@uth.tmc.edu; zqllc8@fimmu.com

†Equal contributors

${ }^{4}$ School of Biomedical Informatics, The University of Texas Health Science Center at Houston, Houston, TX 77030, USA

${ }^{1}$ Department of Pathology, Nanfang Hospital, Southern Medical University, Guangzhou 510515, China

Full list of author information is available at the end of the article
}

samples and generate the data, from which the findings have greatly improved our understanding of the molecular basis of cancers; these efforts include genomic profiling analysis of cancer such as large-scale genome sequencing projects [4-6]. The Cancer Genome Atlas (TCGA), one of the largest cancer-related genome analysis projects, contributed many impellent effects to the understanding of the underlying genetics of CRC, such as mutation characteristics and copy number alterations [7-9]. Moreover, there were several genome-wide analyses which greatly contributed to the comprehensive profiling of CRC whose results provided significant evidence for the association between loci or genes and 
CRC. These included single nucleotide polymorphisms (SNPs) in genes encoding SMAD7, laminin gamma 1, Tbox 3, cyclin D2, etc. [10-13]. These studies have demonstrated that there are many genetic and epigenetic alterations in one or several processes simultaneously. Although these findings seemed not so systematical to reveal an intuitive concept for the biological process of $\mathrm{CRC}$, it provided a hint that a comprehensive method should be used to uncover the underlying regulation mechanism of these bio-molecules.

Network analysis, such as feedback loop (FBL) and feed-forward loop (FFL), is a powerful way to investigate the underlying global topological structures of molecular networks [14-17]. miRNA-transcription factor (TF) coregulation is one of the important FFL type. Building and mining miRNA-TF co-regulation networks served as a valuable approach to investigate the cell regulation in many systems and cell types, including various kinds of cancers [17-19]. miRNAs are evolutionarily conserved, endogenous, small, and noncoding RNAs molecules of about 22 nucleotides in length. miRNAs play important roles in post-transcriptional gene regulation during the initiation and progression of human cancers [20-23]. A spectrum of dysregulated miRNAs were also identified between CRC and normal colorectal tissues [24]. For example, over expression of miR-20a and weak expression of miR-133b have been consistently reported in CRC versus normal tissues, and play crucial roles in both metastasis and survival [25-28]. TFs regulate gene expression through translating cis-regulatory codes into specific gene-regulatory events. Accompanied with miRNAs, TFs participate in the regulatory network that controls thousands of mammalian genes [14]. Through the co-regulation model, miRNA and TF regulate their mutual target genes: miRNAs regulate gene's posttranscription through binding the 3' untranslated region (UTR) while TFs regulate gene's transcriptions through binding to the gene's promoter region [29]. Additionally, TF can regulate miRNA, or to be regulated by miRNA, so that the relationships among miRNAs and TFs and their shared targets form a diversity of feed-forward loops (FFL) [14]. The typical mixed FFL motif defined as a 3-node FFL consists of three components: TF, miRNA and their mutual regulated gene. Recently, FFL-based combinatorial regulatory network approach has emerged as a promising tool to elucidate complex diseases, such as schizophrenia [30], glioblastoma multiforme [31, 32], ovarian cancer [33], lung cancer [34], and osteosarcoma [35]. However, network based on 3-node FFLs has not been established in CRC, one of the common cancers.

In this study, we investigated the comprehensive miRNA-TF co-regulatory network in CRC through modifying the well-developed framework in our previous studies [32, 33]. Among the candidate genes, we identified the potential targets of CRC-related TFs and miRNAs, then built a comprehensive CRC-specific miRNA-TF mediated regulatory network. Finally, we divided this massive network into three subnetworks on the basis of their inside regulatory relationships, followed by a topology analysis. However, such regulations might include some false positives due to the limitation to recent regulatory prediction databases.

The TCGA studies generated vast quantities of gene expression profiling and other molecular profiling from hundreds of CRC samples, which provide the promising opportunity to uncover the basic building blocks of regulatory networks in CRC [9]. Thus, compared to our previous methods [32,33], we took the advantage of the gene and miRNA expression data in CRC patients from TCGA project to improve the accuracy of the results $[7,9]$. This integration with experimental data from patients is a complement to the FFL studies which mostly relied on the predicted regulation information by reducing false positives. After these systematic analysis, we identified six hub components. To verify the implication of these components, we further explored the associations between the expression level of identified components and CRC survival. This study established a valuable CRC progress regulation network, which can provide information about further experimental exploration and help to reveal the complicated regulatory mechanisms and find out new markers or targets for the diagnoses and treatments for CRC.

\section{Methods}

\section{CRC-related genes and miRNAs}

We collected CRC-related genes from five sources (Fig. 1). These sources included the Cancer Gene Census (CGC, available at [36]), the Online Mendelian Inheritance in Man (OMIM, available at [37]), The Cancer Genome Atlas (TCGA) publication [9] and its mutation data (available at [38]), and a mutation landscape research [39]. Finally 464 unique genes were obtained (Additional file 2: Table S1 and Additional file 3: Text S1).

To obtain the dysregulated miRNAs in CRC, we searched the miR2Disease (available at [40]), PhenomiR2.0 (available at [41]), and HMDD2.0 (available at [42]) by using the keywords "colorectal cancer" or "colorectal neoplasms or colonic neoplasms". The expressions of miRNAs obtained from miR2Disease and PhenomiR2.0 have already been recorded. For HMDD2.0, we downloaded the full papers through the related PubMed ID and read those texts to identify the expression comparison between CRC and normal controls. Finally, 257 unique miRNAs were retrieved as CRC-related miRNAs (Additional file 2: Table S2 and Additional file 3: Text S2). 


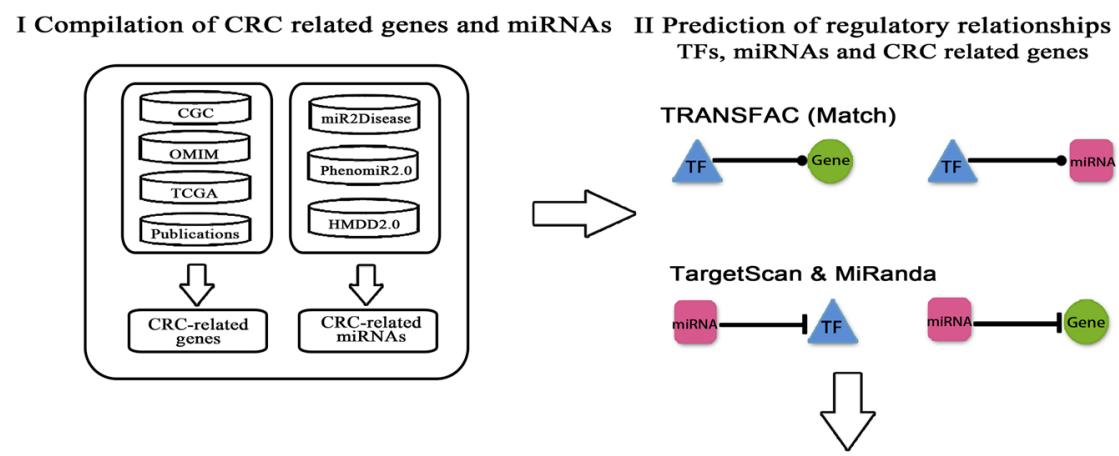

IV construction of the regulatory network

III Identification of significant FFLs

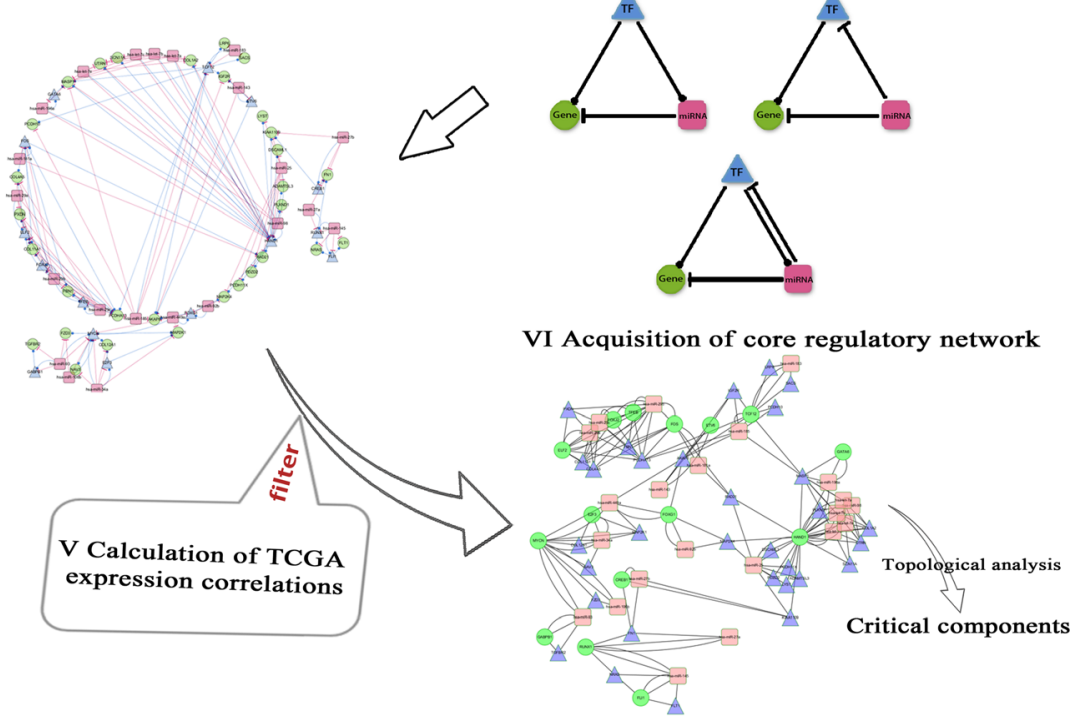

Fig. 1 Process of miRNA-TF regulatory network construction and significant FFLs identification in colorectal cancer (CRC). This process contains six steps. 1) Data compilation. We extracted CRC-related genes, CRC-related microRNAs (miRNAs), and human transcription factors (TFs) from multiple databases. 2) Prediction of the regulatory relationships. The four regulatory relationships include TF-gene, TF-miRNA, miRNA-gene, miRNA-TF. 3) Feed-forward loop identification. Based on the regulatory relationships above, the significant 3-node feed-forward loops were identified. 4) CRCspecific miRNA-TF regulatory network construction and further analysis by merging the FFLs identified in step three. 5) TCGA expression correlation calculation. We calculated the expression correlations of each pair in the network, and removed the false positive pairs. 6) Acquisition of significant FFLs. We extracted the core subnetwork based on the significant pairs identified in step five. Furthermore, identification of critical miRNA and gene components were performed

\section{Prediction of the regulatory relationships}

We applied the TargetScan and the miRanda to obtain the regulatory relationship between miRNAs and CRC-related genes or human TFs. We downloaded the TargetScan database (Release 6.2, available at [43]) and extracted the miRNA-gene pairs. These pairs are evolutionarily conserved in the four species (include human, mouse, rat and dog) and have a total context score higher than -0.30 . For miRanda (available at [44]), we extracted the target pairs conserved in human, mouse and rat with the condition of $S>90$ and $\Delta G<-17$. Then we merged the two sets of miRNA-gene pairs together. To obtain the regulation of miRNA to TF, we retrieved 1201 TFs from the TRANSFAC Professional
Database (release 2011.4) [45]. We extracted the TFs based on its CRC-related target promoter region sequences $(-1500 /+500$ around TSS). Then we performed a binding sites search of TFs to the defined promoter region of the $\mathrm{CRC}$-related targets. Then we used pre-calculated cut-offs to minimize false positive (minFP) matches and created a high-quality matrix. To restrict the search, we required a core score of 1.00 , a matrix score of 0.95 , and TF that only belong to the human genome. To further reduce false positive prediction, we required the predicted pairs to be conserved among humans, mice and rats. For the regulation of TF to genes/miRNAs, we followed the procedure we utilized in our previous work [32]. 


\section{Selection of significant regulations based on TCGA expression data}

The Cancer Genome Atlas (TCGA) project provides a large data to the cancer research. We first downloaded the CRC-related expression data from the TCGA Data Portal (available at [38]), and calculated the correlation among the gene and miRNA nodes of the regulatory networks. Significant pairs were selected on the basis of the expression Pearson correlation coefficient (R). For TF-gene pairs, we required $R \geq 0.14$ or $R \leq-0.14$ (adjusted $P$-value $<0.01$, adjusted by FDR, one-tailed probability, sample size $=264$ ). For miRNA-gene pairs, we required $R \leq-0.15$ (adjusted $P$-value $<0.01$, adjusted by FDR, one-tailed probability, sample size $=243$ ). For TFmiRNA pairs, we required $R \geq 0.15$ or $R \leq-0.15$ (adjusted $P$-value $<0.01$, adjusted by FDR, one-tailed probability, sample size $=243$ ). For miRNA-TF pairs, we required $R \leq-0.15$ (adjusted $P$-value $<0.01$, adjusted by FDR, one-tailed probability, sample size $=243$ ).

Significant component expression and survival correlation analysis

Expression and survival data was obtained from the OncoLnc database, available at [46]. The optimum cutoff level of expression of each component was selected on the basis of the association with the patients' survival by using a tool X-tile (version 3.6.1). A log-rank test was used to compare survival curves.

\section{Network visualization and data analysis}

We visualized the network by using Cytoscape (version 3.2.0) [47]. All statistical analyses were performed with $R$ software and appropriate packages, available at [48].

\section{Results}

Regulatory relationships among miRNAs, TFs, and genes To build miRNA-TF co-regulatory networks in CRC, we modified the computational framework developed in our previous studies (Fig. 1). In the process, the 464 CRCrelated genes with mutation evidence from five data sources (Additional file 2: Table S1 and Additional file 3: Text S1), the 257 miRNAs that reported to be dysregulated in the CRC (Additional file 2: Table S2 and Additional file 3: Text S2), and the $1201 \mathrm{TFs}$ from TRANSFAC Professional (release 2011.4) [49] were collected. 1201 TFs were not preselected based on other evidences related to $\mathrm{CRC}$, but filtered out by strict requirements when identified regulatory (see Methods). Four types of regulatory relationships among genes, miRNAs and TFs were predicted by using the methods described in our previous study [32]. Prediction results of the regulatory relationships were summarized in Table 1. These predicted relationships were named as prediction data.

\section{CRC-specific regulatory networks generated from prediction data}

By merging the regulatory relationships predicted above, 3-node FFLs were formed (Table 2). The 3-node FFL, as one of the most common types of motifs in transcriptional network, can be classified into three categories: miRNA-FFL, TF-FFL and composite FFL, which are based on their inside regulations and have been described in our previous study [32]. In general, in miRNA-FFL, the miRNA represses both TF and gene expression while the TF regulates target gene expression. In TF-FFL, the TF regulates the miRNA and the gene while the miRNA represses the target gene. In composite-FFL, the TF regulates the miRNA and target gene while the miRNA represses the TF and the gene. The three types of FFLs are exclusive to each other.

A miRNA-TF mediated network was constructed for CRC based on 3-node FFLs obtained above. The network contained 12,821 edges and 312 unique nodes of the 13,123 FFLs (Additional file 2: Table S3). Among the 12,821 edges, 174 were miRNA-gene pairs, 57 were miRNA-TF pairs, 7043 were TF-gene pairs, and 5547 were TF-miRNA pairs. Among the 312 nodes, 82 were CRC-related genes, 59 were CRC-related miRNAs, and 171 were human TFs. Considering that these FFLs could

Table 1 Regulatory relationships among CRC-related genes, CRC-related miRNAs and TFs

\begin{tabular}{|c|c|c|c|c|c|}
\hline Relationship & Number of pairs & Number of miRNAs ${ }^{a}$ & Number of genes & Number of TFs ${ }^{b}$ & Method \\
\hline miRNA-gene ${ }^{c}$ & 201 & 60 & 91 & - & TargetScan and miRanda \\
\hline miRNA-TF ${ }^{d}$ & 106 & 58 & - & 43 & Match $^{\text {TM }}$ \\
\hline TF-gene $e^{e}$ & 42,023 & - & 401 & 189 & Match $^{\mathrm{TM}}$ \\
\hline TF-miRNA ${ }^{f}$ & 25,109 & 234 & - & 189 & Match $^{\mathrm{TM}}$ \\
\hline Total & 67,439 & 235 & 410 & 189 & - \\
\hline
\end{tabular}

amiRNA: microRNA

${ }^{\mathrm{b}}$ TF: transcription factor

cmiRNA-gene: miRNA repression of gene expression

${ }^{d}$ miRNA-TF: miRNA repression of gene expression

'TF-gene: TF regulation of gene expression

${ }^{\mathrm{f}}$ TF-gene: TF regulation of miRNA expression 
Table 2 Summary of 3-node feed-forward loops based on CRC-related prediction data

\begin{tabular}{|c|c|c|c|c|c|c|c|c|c|}
\hline \multicolumn{5}{|c|}{ Number of nodes ${ }^{a}$} & \multicolumn{5}{|c|}{ Number of links } \\
\hline 3-node motif & Number of merged FFLs ${ }^{\mathrm{b}}$ & Genes & miRNAs & $\overline{\mathrm{TFS}}$ & $\overline{\text { Total }}$ & TF-gene & miRNA-gene & miRNA-TF & $\overline{\text { TF-miRNA }}$ \\
\hline TF-FFL & 13,005 & 82 & 59 & 170 & 12,680 & 7001 & 174 & 0 & 5505 \\
\hline miRNA-FFL & 25 & 20 & 13 & 12 & 61 & 23 & 23 & 15 & 0 \\
\hline Composite-FFL & 93 & 42 & 30 & 24 & 225 & 64 & 77 & 42 & 42 \\
\hline Total & 13,123 & 82 & 59 & 171 & 12,821 & 7043 & 174 & 57 & 5547 \\
\hline
\end{tabular}

${ }^{a}$ Definition of the nodes and links is the same as in Table 1

${ }^{\mathrm{b}} \mathrm{FFL}$ : feed-forward loop

be categorized into miRNA-FFLs, TF-FFLs, and composite-FFLs, three subnetworks consisted of corresponding type of FFL were generated accordingly. We named them miRNA-SNW, TF-SNW, and compositeSNW, respectively (Fig. 2). To provide a general view of them, we calculated the degrees and their distributions in all the three subnetworks [50].

The miRNA-SNW composed of 25 (25 out of 13,123, 0.19\%) miRNA-FFLs containing 61 edges and 45 individual nodes (Fig. 2a and Additional file 2: Table S4). Among the 61 edges, 23 were miRNA-gene pairs, 15 were miRNA-TF pairs, and 23 were TF-gene pairs. Among the 45 nodes, 20 (20 out of 82, 24.39\%) were CRC-related genes, 13 (13 out of 59, 22.03\%) were CRC- related miRNAs, and 12 (12 out of $171,7.02 \%$ ) were human TFs. The degree values for genes, miRNAs, TFs in this network were in the range of $2-4,2-7$, and $2-7$, respectively. Especially, the degree distribution for miRNAs was strongest right-skewed. The distribution pointed out that most of the nodes had low degrees (less than or equal to 3 ), while only a small portion of them had high degrees. There was only one miRNA hsa-miR25 had a high degree value (the degree value was 7) (Fig. 2 and Additional file 2: Table S5). This distribution analysis uncovered that hsa-miR-25 regulated more targets than any other regulators.

The TF-SNW was consisted of 12,680 edges and 311 unique nodes from 13,005 (13,005 out of 13,123,

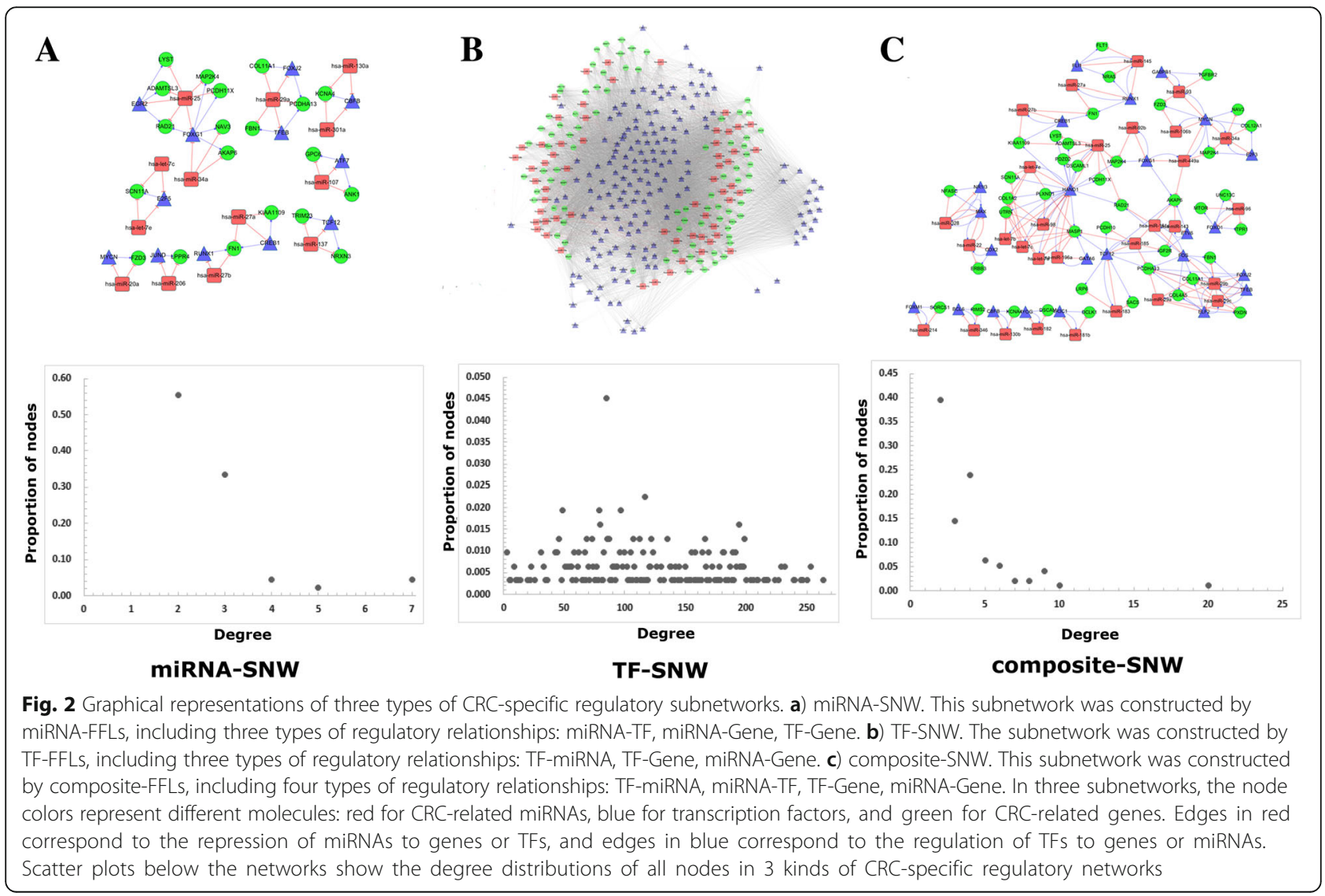


99.10\%) TF-FFLs (Fig. 2b and Additional file 2: Table S4). Among the 12,680 edges, 174 were miRNA-gene pairs, 7001 were TF-gene pairs, and 5505 were TFmiRNA pairs. Among the 311 nodes, 82 (82 out of 82, $100 \%$ ) were CRC-related genes, 59 (59 out of 59, 100\%) were CRC-related miRNAs, and 170 (170 out of 171, 99.42\%) were human TFs. The degree values of genes, miRNAs, TFs ranged from 44 to 191,97 to 264 , and 3 to 200 , respectively. However, their degrees followed a normal distribution. This means that there were few extreme values and was not as helpful as the other two subnetworks for finding biologically critical nodes (Fig. 2 and Additional file 2: Table S5).

In the composite-SNW, there were 93 (93 out of 13,123, $0.71 \%$ ) composite-FFLs, 96 unique nodes, and 225 edges (Fig. 2c and Additional file 2: Table S4). Among the 225 edges, 77 were miRNA-gene pairs, 42 were miRNA-TF pairs, 64 were TF-gene pairs, and 42 were TF-miRNA pairs. Among the 225 nodes, 30 (30 out of 59,50.85\%) were CRC-related miRNAs, 42 (42 out of $82,51.22 \%$ ) were CRC-related genes, and 24 (24 out of 171, 14.04\%) were human TFs. The result showed that the composite-FFLs occupied pretty low proportion of all the FFLs, while recruited more than half of CRC-related genes and miRNAs. This indicated that the composite-FFLs might play more important roles than the other two kinds of FFLs. In this subnetwork, degree values of genes, miRNAs and TFs ranged from 2 to 10,2 to 9 , and 2 to 20 , respectively. The gene that had the largest degree was MASP1; and the miRNA and TF having the largest degrees were hsa-miR-25, hsa-miR-29b and HAND1 respectively (Fig. 2 and Additional file 2: Table S5).

Among above three subnetworks, 15 genes (FZD3, KCNA4, RAD21, KIAA1109, LYST, SCN11A, AKAP6, PCDHA13, ADAMTSL3, PCDH11X, MAP2K4, COL11A1, FBN1, NAV3 and FN1), 7 miRNAs (hsa-miR25, hsa-miR-29a, hsa-miR-34a, hsa-let-7c, hsa-let-7e, hsa-miR-27b, hsa-miR-27a) and 8 TFs (FOXG1, TCF12, FOXJ2, MYCN, TFEB, CREB1, RUNX1, CBFB) participated in all subnetworks simultaneously, which suggested that they might act extensively in the CRC regulation. Interestingly, we noticed that hsa-miR-25 had the highest degree value in both of the composite-SNW and miRNA-SNW, suggesting that hsa-miR-25 might be a critical molecule in the regulatory process of CRC.

\section{CRC-specific significant regulatory network generated by integrating TCGA expression data}

The network generated above was systematical and comprehensive, but it was too complicated to explore the specific regulation mechanisms in CRC. To obtain the regulatory relationship with higher accuracy, we took the advantage of the gene and miRNA expression data in CRC patients from TCGA. Firstly, the correlation coefficients among genes, TFs, and miRNAs were calculated, and then stringent constraint conditions (see Methods) were required to define a co-expression. Subsequently, four types of links (miRNA-gene, miRNA-TF, TF-gene, and TF-miRNA) were obtained (Table 3). We named the dataset Experiment_data that included all these pairs based on TCGA experimental data.

To reduce the false positives, pairs (regulatory relationships) were required to be conserved in both the prediction data and Experiment_data. Finally, one composite-FFL (hsa-miR-25, HAND1, ADAMTSL3), one miRNA-FFL (hsa-miR-25, EGR2, ADAMTSL3) and 56 TF-FFLs were identified. The regulation details are presented in Fig. 3 and Additional file 1: Figure S1. The number of TF-FFL was significant more than the other two. In these TF-FFLs, there were 115 edges ( $55 \mathrm{TF}$-gene pairs, 53 TF-miRNA pairs, and 7 miRNA-gene pairs) and 58 unique nodes ( 45 human TFs, 7 CRC-related genes, and 6 CRC-related miRNAs) Additional file 2: Table S6). There are a few nodes exhibited a high degree, which acted as the hubs that might play more important roles in the regulatory networks [51, 52]. Using the hub definition method proposed by $\mathrm{Yu}$ et al. [53], we determined the degree cutoff value of 22, 26, and 7 for gene, miRNA and TF hubs respectively (Additional file 2: Table S7). Accordingly, two hub miRNAs (hsa-miR-25 and hsa-miR-31), two hub genes (ADAMTSL3 and AXIN1) and one hub TF (BRCA1) were identified.

Table 3 Summary of co-expression relationships among CRC-related genes, CRC-related miRNAs, and TFs from TCGA

\begin{tabular}{lllll}
\hline Co-expression relationship & Number of pairs & Number of miRNAs & Number of genes & Number of TFs $^{\mathrm{b}}$ \\
\hline miRNA-gene $^{c}$ & 550,079 & 567 & 19,570 & - \\
miRNA-TF $^{\mathrm{a}}$ & 28,563 & 553 & - & 1141 \\
TF-gene $^{\mathrm{e}}$ & $1,126,334$ & - & 19,701 & 1158 \\
TF-miRNA $^{\mathrm{f}}$ & 60,794 & 570 & - & 1201 \\
\hline
\end{tabular}

amiRNA: microRNA

${ }^{\text {bTF: }}$ transcription factor

CmiRNA-gene: anti-correlation between miRNA and gene expression

${ }^{d}$ miRNA-TF: anti-correlation between miRNA and TF expression

eTF-gene: correlation between TF and gene expression

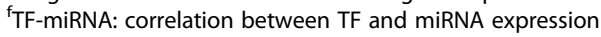




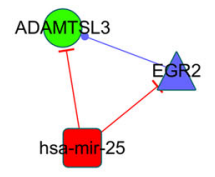

miRNA-FFLs

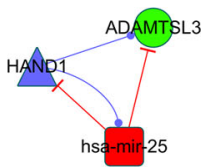

composite-FFLs

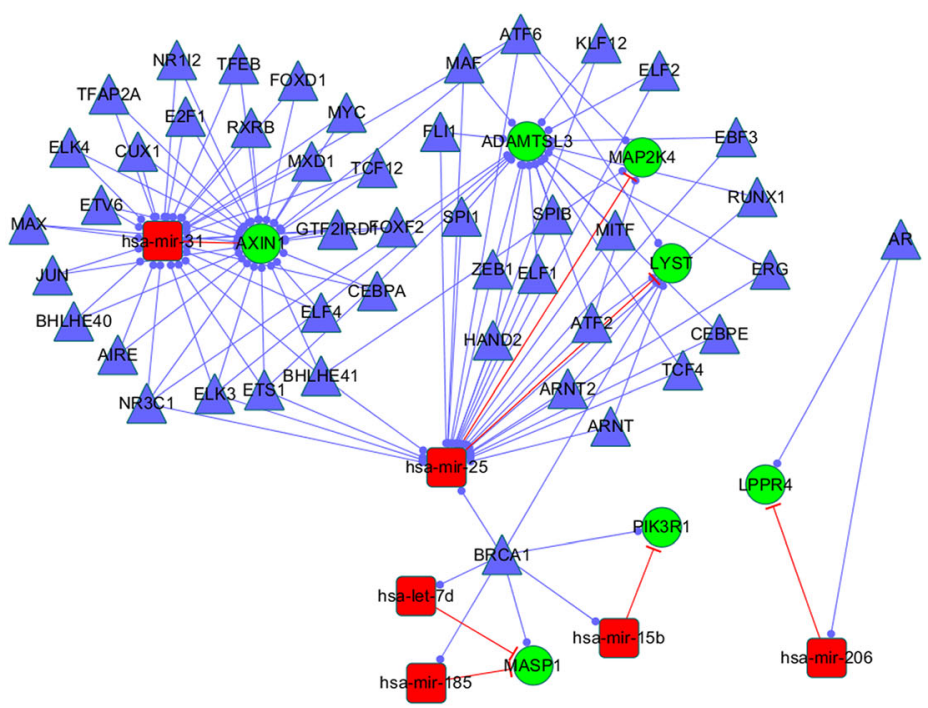

TF-FFLs

Fig. 3 Graphical representation of the significant FFLs. The regulatory network was generated from 3-node FFL motifs common to the prediction data and Experiment_data. Shapes and colors definitions for nodes and edges are the same as in the Fig. 2

\section{Significant components}

As analyzed above, through our consecutive network framework, 5 components were identified, including two hub miRNAs (hsa-miR-25 and hsa-miR-31), two hub genes (ADAMTSL3 and AXIN1) and one hub TF (BRCA1). Such hub identification was mainly based on their degrees in the network. Are these connective characteristics specific to CRC, or just their innate property of the complex regulatory mechanism in our body? We found hsa-miR-25 had more targets (top 5.0\%, Additional file 2: Table S8) than most of others miRNAs collected in TargetScan but less targets in miRanda (top $60.0 \%$, Additional file 2: Table S9), and hsa-miR-31 had a moderate number of target in both databases (top 36.8\% and $32.8 \%$, respectively). However, some miRNAs, such as hsa-miR-7b and hsa-miR-497, had a high number of targets both in TargetScan (top 4.0\% and 0.8\%, respectively) and miRanda (top $6.0 \%$ and $11.2 \%$, respectively), which were also included in our analysis, but they were not identified as hub nodes after our consecutive analysis. These suggested that the significant miRNA identification was mainly contributed to the regulatory pattern after our regulatory network construction, despite of the relationship distribution and bias in databases might make an impact on the topology of final network.

To further investigate the implication of the hub miRNAs, TFs and genes for CRC development, we analyzed the correlation between their expression levels and survivals of patients with CRC by using data from OncoLnc database [46]. Figure 4 shows the expression of the significant components in CRC patients with low or high risk to all-caused dead and the survival curves in the low and high risk groups which were identified by the optimal cut-off value of corresponding component expression level. All of the significant components showed a well prediction value for the prognosis of CRC patients. Among 5 significant components, hsa-miR-25, AXIN1, ATF6 and BRCA1 exhibited a negative correlation between their expression levels and patients' survival, while higher expression of ADAMTSL3 was observed in patients with a better survival. Patients was subdivided well into two groups (namely, low risk and high risk groups) by using these components independently, with significantly different survival curves.

\section{Discussion}

In this study, a co-regulatory network mediated by miRNAs and TFs was first time explored in CRC, one major cancer type. Our results provides some insightful information and a few miRNA and TF candidates, as well as their regulation for further experimental validation in CRC. In this study, our previous computational framework was modified by integrating gene and miRNA expression data from TCGA to improve the result accuracy. We extracted significant components from the whole complex network based on prediction data by using the data of Experiment_data. Then survival information was used to determine the significant components implication for CRC prognosis.

This unique computational framework has been described in our previous studies [32, 33] and illustrated that it is indeed possible to use a large panel of methods to process multiple types of data (e.g., mutation data, gene expression data, and knowledgebase) to identify 


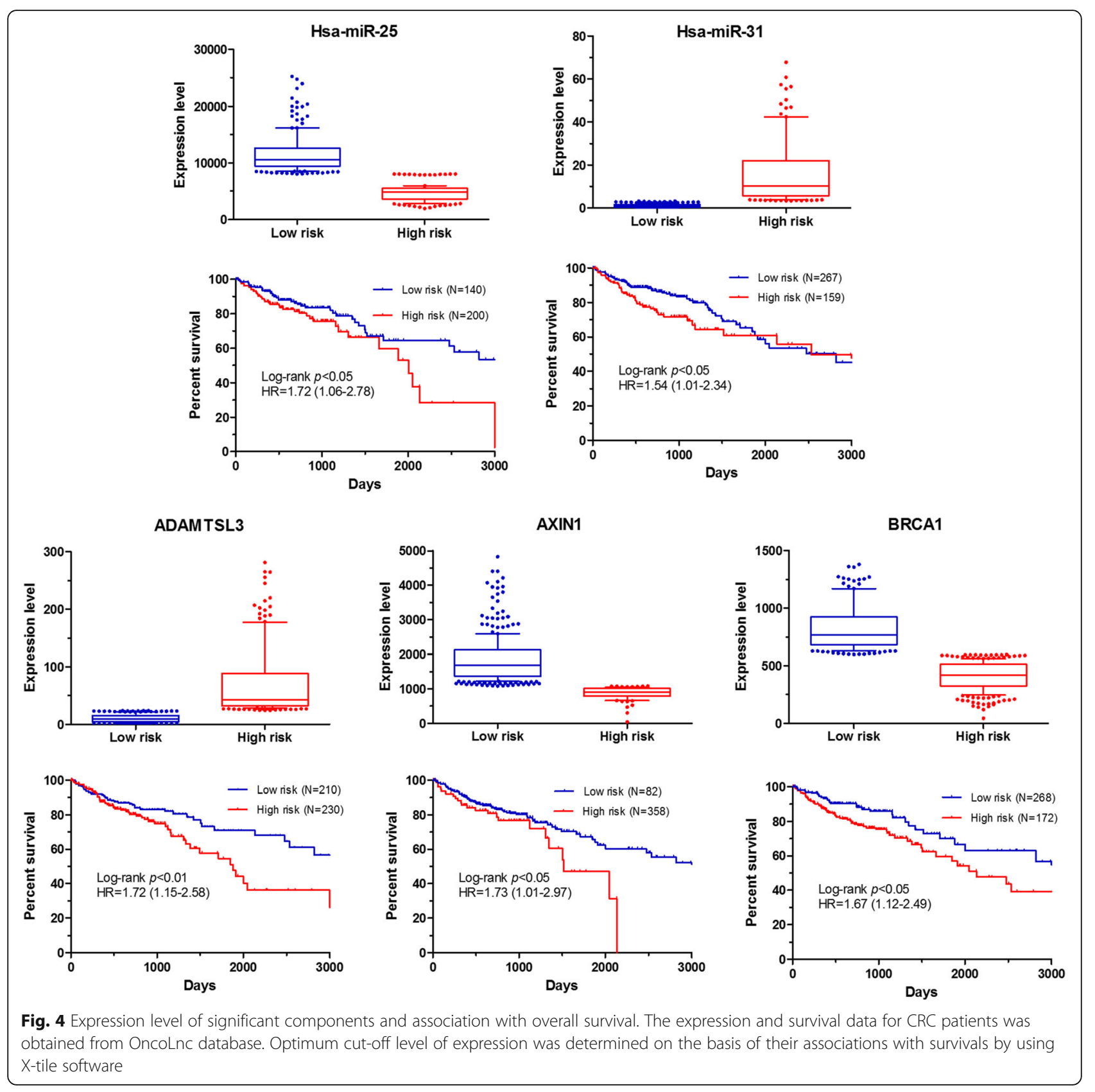

potential disease-associated components in complex diseases. To increase the confidence and accuracy in predicting biologically relevant regulations, one strategy is to identify regulatory relationships that are consistent or reproducible in multiple independent studies $[54,55]$. In this study, as the major improvement for our previous computational framework, we specifically integrate the prediction data and experiment data in our regulatory network analyses. The experiment data was used to improve the accuracy of results in the prediction data, whereby the significant components were extracted from the whole huge and complex network. So far, such a strategy has not been applied to miRNA-TF coregulatory network analyses in CRC. Furthermore, with the rapid growth in high-throughput expression profiling studies, this strategy might become not only feasible, but also necessary to identify complex gene regulation in cellular systems and provides a supplement for regulatory network investigation.

Using the prediction data, a massive and complex network was built for CRC, which could be subdivided into 3 exclusive subnetworks, namely composite-SNW, miRNASNW, TF-SNW. We found that some components participated in three types of subnetworks simultaneously, 
including 15 genes (FZD3, KCNA4, RAD21, KIAA1109, LYST, SCN11A, AKAP6, PCDHA13, ADAMTSL3, PCDH11X, MAP2K4, COL11A1, FBN1, NAV3 and FN1), 7 miRNAs (hsa-miR-25, hsa-miR-29a, hsa-miR-34a, hsa-let7c, hsa-let-7e, hsa-miR-27b, hsa-miR-27a) and 8 TFs (FOXG1, TCF12, FOXJ2, MYCN, TFEB, CREB1, RUNX1, CBFB). In this study, we aimed to find out some significant components (miRNA, gene, or TF), which could serve as biomarker for the diagnosis, treatment, and prognosis of CRC. Although there were some interesting findings in the predictive network, it was difficult and unconvincing to determine significant components for the two reasons. First, the networks involved a great many components, especially TF-SNW, the regulations were massive and complex. Second, since the regulations involved in current networks were on the basis of multiple data sources, not all of which was validated by experiments, there might be some false positives. To improve our network, we integrated the expression data from TCGA into our analysis and used the co-expression to wash the unreliable regulations in the network. We then applied the hub identification to the concise network to determine significant components, whereby two miRNAs (hsa-miR-25 and hsa-miR-31), two genes (ADAMTSL3 and AXIN1) and one TF (BRCA1) were identified significantly. Some of those genes, miRNAs and TFs have been reinforced by previous studies. To investigate values of these components on prognosis, we further analyzed association between their expression levels and survivals. We found that all of five components showed a promising predictive ability for CRC patients' survival. For instance, low expression of hsa-miR-25 was observed with the increasing all-caused death risk for CRC patients. This is consistent with previous reports. In Li's study, miR-25 was found to be down-regulated in human colon cancer tissues when compared to those in matched non-neoplastic mucosa tissues [56]. Functional studies revealed that restoration of miR-25 expression inhibited cell proliferation and migration. In contrast, miR-25 inhibition could promote the proliferation and migratory ability of cells. Stable overexpression of miR-25 also suppressed the growth of colon cancer-cell xenografts in vivo [57]. In Koo BH's study, identification of frequent ADAMTSL3 mutations in colorectal cancer suggested it might have a regulatory role in cellular homeostasis in colorectal epithelium or in pathways to colorectal malignancy [58]. In current study, the expression level ADAMTSL3 was found correlated with all-caused survival. Approximately half of the genes, miRNAs, TFs we predicted to be key roles had been studied and found to be associated with the regulation mechanism in CRC. These results indicated that the comprehensive CRC-specific regulatory network could provide valuable clues for researchers to identify critical CRC-related components. Furthermore, as hsa-miR-25 and ADAMTSL3 had been proved playing important roles in CRC, but their exact interaction mechanism have not been clarified yet. Other significant components identified in our analysis also remain unclarified and need to explore by further researches.

A recent study by $\mathrm{Fu}$ et al. used a combinatorial strategy to identify CRC-related miRNA-mRNA pairs [59]. This study applied microarray expression data to identify dysregulated miRNAs and mRNAs, followed by anti-correlation computation and target relationship prediction based on TargetScan and miRanda. 72 miRNA-mRNA pairs were captured by including 22 miRNAs and 58 mRNAs. But these results were only limited in the binary regulation model between miRNAs and mRNAs, and the sample size of study was small (8 pairs). Although several studies aiming to uncover the regulation system of TFs and miRNAs have been reported [59-61], none have considered the integration of predictive data and experimental data in the application of an FFL model in CRC, improving the stability and reliability of the regulatory network. The process in current study could be a useful method and complement for revealing the complex regulation in other disease.

There also exist several limitations to our analysis. First, the number of relationship and its collective bias in the databases might make a potential effect on the final network construction and following significant components identification. In our analysis process, data selection were performed by multisource to reduce such impact. Second, as opposed to gene and miRNA, TF was not pre-selected to be CRC-related, which might influence the topology observation. In addition to the criteria used in regulation prediction in current study, more effective selection need to apply to CRC-related TF identification.

\section{Conclusions}

Recently, network analyses have been applied to many diseases to reveal the complicated mechanisms and try to find out new makers or targets for the diagnoses and treatments. However, network analysis have not been systematically applied in colorectal cancer (CRC). In our paper, we build a systematic, comprehensive and complicated network for $\mathrm{CRC}$, and finally through topologic analysis, we find some key miRNAs and feed forward loops that possibly play important roles in the regulation of CRC for further experiment design.

Furthermore, current FFL studies mostly rely on the predicted regulation information, which may lead to false positive outcomes. So some strategies are urgently needed to reduce the false positive rate. In this field, we integrated the predictive information and experimental co-expression data of TCGA project. We finally extracted significant components for CRC from a comprehensive and complex network using this strategy, which was confirmed in the subsequent prognosis analysis. This innovative strategy can be an inspiration for further researches in this field. 


\section{Additional files}

Additional file 1: Figure S1. Shows the degree distributions of nodes in the significant FFLs. (ZIP $289 \mathrm{~kb}$ )

Additional file 2: Tables S1 through S9. Table S1. Shows the CRCrelated genes compiling from four sources. Table $\mathbf{S 2}$. shows the CRCrelated miRNAs compiling from three sources. Table S3. shows merged 3node FFLs including TF-FFLs, miRNA-FFLs and composite-FFLs. Table S4. shows the regulation information of the CRC-specific miRNA-TF mediated regulatory network. Table S5. shows the degree distribution of all nodes in the miRNA-SNW, TF-SNW and composite-SNW. Table S6. shows the regulation information of the CRC-specific significant FFLs. Table S7. shows the degree distribution of all nodes in the CRC-specific significant FFLs. Table S8. shows the miRNA targets predicted by using TargetScan. Table S9. shows the miRNA targets predicted by using miRanda. (ZIP 62739 kb)

Additional file 3: Texts S1 though S2. TextS1. Compiles CRC-related genes from multiple datasets. Text S2. compiles CRC-related miRNAs from multiple datasets. (ZIP $9 \mathrm{~kb}$ )

\section{Abbreviations}

CRC: Colorectal cancer; FBL: feedback loop; FFLs: feed-forward loops; miRNA: microRNA; SNPs: single nucleotide polymorphisms; SNW: subnetwork; TCGA: The Cancer Genome Atlas; TFs: transcription factors

\section{Acknowledgements}

Not applicable.

\section{Funding}

This work was supported in part by grants from National Natural Science Foundation of China (\#81472712 and \#81071989) and Guangdong Science and Technology Department (\#C1221020700008). Dr. Zhao was partially supported by NIH grant R21CA196508.

\section{Availability of data and materials}

All data generated or analyzed during this study are included in this published article [and its supplementary information files].

\section{Authors' contributions}

Oversight and leadership responsibility for the research activity planning and execution, including mentorship external to the core team: JS QZ. Management and coordination responsibility for the research activity planning and execution: JS QZ. Acquisition of the financial support for the project leading to this publication: JS QZ ZZ. Conceptualization: JS QZ HW JL CL HN YD. Development or design of methodology: JS QZ HW JL CL. Software: HW JL CL. Validation: JS QZ YD HN. Application of statistical, mathematical, computational techniques to analyze study data: HW JL CL QL JW ZZ HX YD HN. Preparation and presentation of the published work: all authors. Revise and approve the final manuscript: all authors.

\section{Ethics approval and consent to participate}

Not applicable.

\section{Consent for publication}

Not applicable.

\section{Competing interests}

The authors declare that they have no competing interests.

\section{Publisher's Note}

Springer Nature remains neutral with regard to jurisdictional claims in published maps and institutional affiliations.

\section{Author details}

'Department of Pathology, Nanfang Hospital, Southern Medical University, Guangzhou 510515, China. ${ }^{2}$ Department of Pathology, College of Basic Medicine, Southern Medical University, Guangzhou 510515, China. ${ }^{3}$ Center for Quantitative Sciences, Vanderbilt University School of Medicine, Nashville, TN 37232, USA. ${ }^{4}$ School of Biomedical Informatics, The University of Texas
Health Science Center at Houston, Houston, TX 77030, USA. ${ }^{5}$ Center for Precision Health, School of Biomedical Informatics, The University of Texas Health Science Center at Houston, Houston, TX 77030, USA.

Received: 22 October 2016 Accepted: 21 August 2017

Published online: 02 September 2017

\section{References}

1. Siegel R, Naishadham D, Jemal A. Cancer statistics, 2013. CA Cancer J Clin. 2013;63(1):11-30.

2. Diciolla A, Cristina V, De Micheli R, Digklia A, Wagner AD. news and perspectives in the treatment of advanced gastric and colorectal cancers. Rev Med Suisse. 2015:11(475):1122. 1124-1126

3. Ioannou M, Paraskeva E, Baxevanidou K, Simos G, Papamichali R, Papacharalambous C, Samara M, Koukoulis G. HIF-1alpha in colorectal carcinoma: review of the literature. J BUON. 2015;20(3):680-9.

4. Beg S, Siraj AK, Prabhakaran S, Bu R, Al-Rasheed M, Sultana M, Qadri Z, AlAssiri M, Sairafi R, Al-Dayel F, et al. Molecular markers and pathway analysis of colorectal carcinoma in the Middle East. Cancer. 2015;

5. Zhang L, Zhang S, Yao J, Lowery FJ, Zhang Q, Huang WC, Li P, Li M, Wang $X$, Zhang $C$, et al. Microenvironment-induced PTEN loss by exosomal microRNA primes brain metastasis outgrowth. Nature. 2015;527(7576):100-4

6. Seki M, Nishimura R, Yoshida K, Shimamura T, Shiraishi Y, Sato Y, Kato M, Chiba K, Tanaka H, Hoshino N, et al. Integrated genetic and epigenetic analysis defines novel molecular subgroups in rhabdomyosarcoma. Nat Commun. 2015:6:7557.

7. Cancer Genome Atlas Research N, Weinstein JN, Collisson EA, Mills GB, Shaw KR, Ozenberger BA, Ellrott K, Shmulevich I, Sander C, Stuart JM. The cancer genome atlas pan-cancer analysis project. Nat Genet. 2013;45(10):1113-20.

8. Neapolitan R, Horvath CM, Jiang X. Pan-Cancer analysis of TCGA data reveals notable signaling pathways. BMC Cancer. 2015;15:516.

9. Network CGA. Comprehensive molecular characterization of human colon and rectal cancer. Nature. 2012:487(7407):330-7.

10. Broderick P, Carvajal-Carmona L, Pittman AM, Webb E, Howarth K, Rowan A, Lubbe S, Spain S, Sullivan K, Fielding S, et al. A genome-wide association study shows that common alleles of SMAD7 influence colorectal cancer risk. Nat Genet. 2007;39(11):1315-7.

11. Peters U, Jiao S, Schumacher FR, Hutter CM, Aragaki AK, Baron JA, Berndt SI, Bezieau S, Brenner $\mathrm{H}$, Butterbach $\mathrm{K}$, et al. Identification of genetic susceptibility loci for colorectal tumors in a genome-wide meta-analysis. Gastroenterology. 2013;144(4):799-807. e724

12. Tomlinson I, Webb E, Carvajal-Carmona L, Broderick P, Kemp Z, Spain S, Penegar S, Chandler I, Gorman M, Wood W, et al. A genome-wide association scan of tag SNPs identifies a susceptibility variant for colorectal cancer at 8q24.21. Nat Genet. 2007;39(8):984-8.

13. Tomlinson IP, Webb E, Carvajal-Carmona L, Broderick P, Howarth K, Pittman AM, Spain S, Lubbe S, Walther A, Sullivan K, et al. A genome-wide association study identifies colorectal cancer susceptibility loci on chromosomes 10p14 and 8q23.3. Nat Genet. 2008;40(5):623-30.

14. Shalgi R, Lieber D, Oren M, Pilpel Y. Global and local architecture of the mammalian microRNA-transcription factor regulatory network. PLoS Comput Biol. 2007;3(7):e131.

15. Tsang J, Zhu J, van Oudenaarden A. MicroRNA-mediated feedback and feedforward loops are recurrent network motifs in mammals. Mol Cell. 2007; 26(5):753-67.

16. Bartel DP, Chen CZ. Micromanagers of gene expression: the potentially widespread influence of metazoan microRNAs. Nat Rev Genet. 2004;5(5): 396-400.

17. Baskerville S, Bartel DP. Microarray profiling of microRNAs reveals frequent coexpression with neighboring miRNAs and host genes. RNA. 2005;11(3):241-7.

18. Cohen EE, Zhu H, Lingen MW, Martin LE, Kuo WL, Choi EA, Kocherginsky M, Parker JS, Chung CH, Rosner MR. A feed-forward loop involving protein kinase Calpha and microRNAs regulates tumor cell cycle. Cancer Res. 2009; 69(1):65-74.

19. O'Donnell KA, Wentzel EA, Zeller KI, Dang CV, Mendell JT. C-Myc-regulated microRNAs modulate E2F1 expression. Nature. 2005:435(7043):839-43.

20. Cho WC. OncomiRs: the discovery and progress of microRNAs in cancers. Mol Cancer. 2007:6:60

21. Abu-Amero KK, Helwa I, Al-Muammar A, Strickland S, Hauser MA, Allingham RR, Liu Y. Screening of the seed region of MIR184 in Keratoconus patients from Saudi Arabia. Biomed Res Int. 2015;2015:604508. 
22. Katayama M, Sjogren RJ, Egan B, Krook A. miRNA let-7 expression is regulated by glucose and TNF-alpha by a remote upstream promoter. The Biochemical journal. 2015;

23. Zhang $Y C, X u Z$ Z, Zhang TF, Wang YL. Circulating microRNAs as diagnostic and prognostic tools for hepatocellular carcinoma. World journal of gastroenterology : WJG. 2015;21(34):9853-62.

24. Lu J, Getz G, Miska EA, Alvarez-Saavedra E, Lamb J, Peck D, Sweet-Cordero A, Ebert BL, Mak RH, Ferrando AA, et al. MicroRNA expression profiles classify human cancers. Nature. 2005;435(7043):834-8.

25. Akcakaya P, Ekelund S, Kolosenko I, Caramuta S, Ozata DM, Xie H, Lindforss U, Olivecrona H, Lui WO. miR-185 and miR-133b deregulation is associated with overall survival and metastasis in colorectal cancer. Int J Oncol. 2011; 39(2):311-8.

26. Brunet Vega A, Pericay C, Moya I, Ferrer A, Dotor E, Pisa A, Casalots A, SerraAracil X, Oliva JC, Ruiz A et al. microRNA expression profile in stage III colorectal cancer: circulating miR-18a and miR-29a as promising biomarkers. Oncol Rep. 2013; 30(1):320-26.

27. Motoyama K, Inoue H, Takatsuno Y, Tanaka F, Mimori K, Uetake H, Sugihara K, Mori M. Over- and under-expressed microRNAs in human colorectal cancer. Int J Oncol. 2009;34(4):1069-75.

28. Xiang KM, Li XR. MiR-133b acts as a tumor suppressor and negatively regulates TBPL1 in colorectal cancer cells. Asian Pacific journal of cancer prevention : APJCP. 2014;15(8):3767-72.

29. Hobert O. Gene Regulation by Transcription Factors and MicroRNAs

30. Guo AY, Sun J, Jia P, Zhao Z. A novel microRNA and transcription factor mediated regulatory network in schizophrenia. BMC Syst Biol. 2010;4:10.

31. Setty M, Helmy K, Khan AA, Silber J, Arvey A, Neezen F, Agius P, Huse JT, Holland EC, Leslie CS. Inferring transcriptional and microRNA-mediated regulatory programs in glioblastoma. Mol Syst Biol. 2012;8:605.

32. Sun J, Gong X, Purow B, Zhao Z. Uncovering MicroRNA and transcription factor mediated regulatory networks in Glioblastoma. PLoS Comput Biol. 2012;8(7):e1002488.

33. Zhao M, Sun J, Zhao Z. Synergetic regulatory networks mediated by oncogene-driven microRNAs and transcription factors in serous ovarian cancer. Mol BioSyst. 2013;9(12):3187-98.

34. Mitra R, Edmonds MD, Sun J, Zhao M, Yu H, Eischen CM, Zhao Z. Reproducible combinatorial regulatory networks elucidate novel oncogenic microRNAs in non-small cell lung cancer. RNA. 2014;20(9):1356-68.

35. Poos K, Smida J, Nathrath M, Maugg D, Baumhoer D, Korsching E. How microRNA and transcription factor co-regulatory networks affect osteosarcoma cell proliferation. PLoS Comput Biol. 2013;9(8):e1003210.

36. The Catalogue Of Somatic Mutations In Cancer (COSMIC) Database. http:// www.sanger.ac.uk/genetics/CGP/cosmic. Accessed 11 Nov 2014.

37. The McKusick's Online Mendelian Inheritance in Man (OMIM) Database. https://omim.org. Accessed 11 Nov 2014.

38. The Cancer Genome Atlas (TCGA) Database. https://gdc.cancer.gov. Accessed 11 Nov 2014

39. Kandoth C, McLellan MD, Vandin F, Ye K, Niu B, Lu C, Xie M, Zhang Q, McMichael JF, Wyczalkowski MA, et al. Mutational landscape and significance across 12 major cancer types. Nature. 2013;502(7471):333-9.

40. The miR2Disease Database. http://www.mir2disease.org. Accessed $25 \mathrm{Sep}$ 2014.

41. The PhenomiR2.0 Database. http://mips.helmholtz-muenchen.de/phenomir. Accessed 25 Sep 2014.

42. The Human MicroRNA Disease Database (HMDD) version 2.0. http://www. cuilab.cn/hmdd. Accessed 25 Sep 2014.

43. The TargetScan Database. http://www.targetscan.org. Accessed 26 Nov 2014.

44. The miRanda Database. http://www.microrna.org. Accessed 26 Nov 2014

45. Dubchak I, Munoz M, Poliakov A, Salomonis N, Minovitsky S, Bodmer R, Zambon AC. Whole-Genome rVISTA: a tool to determine enrichment of transcription factor binding sites in gene promoters from transcriptomic data. Bioinformatics. 2013;29(16):2059-61.

46. The OncoLnc Database. http://www.oncolnc.org. Accessed 24 May 2017

47. Smoot ME, Ono K, Ruscheinski J, Wang PL, Ideker T: Cytoscape 2.8: new features for data integration and network visualization. Bioinformatics 2011, 27(3):431-432.

48. The Comprehensive R ArchiveNetwork. https://cran.r-project.org. Accessed 20 May 2014.

49. Matys V, Kel-Margoulis OV, Fricke E, Liebich I, Land S, Barre-Dirrie A, Reuter I, Chekmenev D, Krull M, Hornischer K, et al. TRANSFAC and its module
TRANSCompel: transcriptional gene regulation in eukaryotes. Nucleic Acids Res. 2006:34(Database issue):D108-10.

50. Barabasi AL, Oltvai ZN. Network biology: understanding the cell's functional organization. Nat Rev Genet. 2004:5(2):101-13.

51. Sun J, Zhao Z. A comparative study of cancer proteins in the human protein-protein interaction network. BMC genomics. 2010;11:Suppl 3-S5.

52. Zotenko E, Mestre J, O'Leary DP, Przytycka TM. Why do hubs in the yeast protein interaction network tend to be essential: reexamining the connection between the network topology and essentiality. PLoS Comput Biol. 2008;4(8):e1000140.

53. Yu H, Greenbaum D, Xin Lu H, Zhu X, Gerstein M. Genomic analysis of essentiality within protein networks. Trends in genetics : TIG. 2004;20(6): 227-31.

54. Langfelder P, Luo R, Oldham MC, Horvath S. Is my network module preserved and reproducible? PLoS Comput Biol. 2011;7(1):e1001057.

55. Dutta B, Pusztai L, Qi Y, Andre F, Lazar V, Bianchini G, Ueno N, Agarwal R, Wang $B$, Shiang $C Y$, et al. A network-based, integrative study to identify core biological pathways that drive breast cancer clinical subtypes. $\mathrm{Br} \mathrm{J}$ Cancer. 2012;106(6):1107-16.

56. Li BQ, Yu H, Wang Z, Ding GH, Liu L. MicroRNA mediated network and DNA methylation in colorectal cancer. Protein and peptide letters. 2013;20(3): 352-63.

57. Li Q, Zou C, Zou C, Han Z, Xiao H, Wei H, Wang W, Zhang L, Zhang X, Tang $\mathrm{Q}$, et al. MicroRNA-25 functions as a potential tumor suppressor in colon cancer by targeting Smad7. Cancer Lett. 2013;335(1):168-74.

58. Koo BH, Hurskainen T, Mielke K, Aung PP, Casey G, Autio-Harmainen H, Apte SS. ADAMTSL3/punctin-2, a gene frequently mutated in colorectal tumors, is widely expressed in normal and malignant epithelial cells, vascular endothelial cells and other cell types, and its mRNA is reduced in colon cancer. International journal of cancer Journal international du cancer. 2007; 121(8):1710-6.

59. Fu J, Tang W, Du P, Wang G, Chen W, Li J, Zhu Y, Gao J, Cui L. Identifying microRNA-mRNA regulatory network in colorectal cancer by a combination of expression profile and bioinformatics analysis. BMC Syst Biol. 2012;6:68.

60. Cui X, He H, He F, Wang S, Li F, Bo X. Network fingerprint: a knowledgebased characterization of biomedical networks. Sci Rep. 2015;5:13286.

61. Sengupta D, Bandyopadhyay S. Topological patterns in microRNA-gene regulatory network: studies in colorectal and breast cancer. Mol BioSyst. 2013;9(6):1360-71.

\section{Submit your next manuscript to BioMed Central and we will help you at every step:}

- We accept pre-submission inquiries

- Our selector tool helps you to find the most relevant journal

- We provide round the clock customer support

- Convenient online submission

- Thorough peer review

- Inclusion in PubMed and all major indexing services

- Maximum visibility for your research

Submit your manuscript at www.biomedcentral.com/submit 